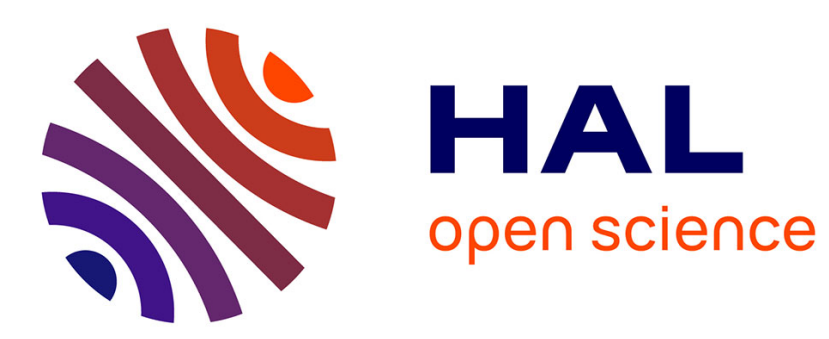

\title{
A global regularisation for integrating the Caledonian symmetric four-body problem
}

\author{
Anoop Sivasankaran, Bonnie A. Steves, Winston L. Sweatman
}

\section{To cite this version:}

Anoop Sivasankaran, Bonnie A. Steves, Winston L. Sweatman. A global regularisation for integrating the Caledonian symmetric four-body problem. Celestial Mechanics and Dynamical Astronomy, 2010, 107 (1-2), pp.157-168. 10.1007/s10569-010-9270-x . hal-00552511

\section{HAL Id: hal-00552511 \\ https://hal.science/hal-00552511}

Submitted on 6 Jan 2011

HAL is a multi-disciplinary open access archive for the deposit and dissemination of scientific research documents, whether they are published or not. The documents may come from teaching and research institutions in France or abroad, or from public or private research centers.
L'archive ouverte pluridisciplinaire HAL, est destinée au dépôt et à la diffusion de documents scientifiques de niveau recherche, publiés ou non, émanant des établissements d'enseignement et de recherche français ou étrangers, des laboratoires publics ou privés. 


\title{
A global regularisation for integrating the Caledonian symmetric four-body problem
}

\author{
Anoop Sivasankaran - Bonnie A. Steves • \\ Winston L. Sweatman
}

Received: 12 November 2009 / Revised: 5 March 2010 / Accepted: 8 March 2010 /

Published online: 28 April 2010

(C) Springer Science+Business Media B.V. 2010

\begin{abstract}
Several papers in the last decade have studied the Caledonian symmetric four-body problem (CSFBP), a restricted four-body system with a symmetrically reduced phase space. During these studies, difficulties have arisen when the system approaches a close encounter. These are due to collision singularities causing numerical integration algorithms to fail. In this paper, we give the full details of a regularisation approach that now enables us to study these close encounters and collision events. The resulting equations of motion can be efficiently integrated by a high-order integrator. The results from numerical testing of the algorithm verify that the regularisation is advantageous in preserving numerical stability. The effectiveness of the approach is illustrated for a range of CSFBP orbits. Numerical experiments show that the newly developed regularisation algorithm has excellent energy conservation properties.
\end{abstract}

Keywords Regularisation · Close encounters · Restricted four-body problem · Caledonian symmetrical four-body problem

\section{Introduction}

This paper discusses the design and verification of an efficient regularisation scheme to study the close encounters that appear in the Caledonian symmetrical four-body problem (CSFBP), a special restricted four-body problem with a symmetrically reduced phase space. This

\footnotetext{
A. Sivasankaran $(\varangle) \cdot$ B. A. Steves

School of Engineering and Computing, Glasgow Caledonian University, Glasgow G4 0BA, UK e-mail: anooppgd@gmail.com

B. A. Steves

e-mail: B.Steves@gcu.ac.uk

\section{W. L. Sweatman}

Institute of Information and Mathematical Sciences, Massey University at Albany,

Private Bag 102904, North Shore, 0745 Auckland, New Zealand

e-mail:w.sweatman@massey.ac.nz
} 
four-body problem has been widely studied in the past, with the help of symmetrical restriction methods which reduce the mathematical complexity of the general few-body problem by reducing the phase space dimensions. Symmetrical restricted four-body problems have been studied recently in Sweatman (2002, 2006), and Sekiguchi and Tanikawa (2004).

The $c^{2} H$ stability criterion proposed in Zare (1976, 1977), and Marchal and Saari (1975), provides a hierarchical stability criterion for the three-body problem. It shows that when the angular momentum $c$ combined with the energy $H$ reaches a critical value $\left[c^{2} H\right]_{\text {crit }}$, the phase space of possible motions can have disconnected topological regions. Loks and Sergysels (1985) and Sergysels and Loks (1987) extended this criterion to the case of the general four-body problem.

Roy and Steves (1998, 2001) and Steves and Roy $(1998,2001)$ developed the CSFBP and derived an analytical stability criterion. These authors showed that the global stability of the system depends on a parameter they called the Szebehely constant $C_{0}$, which is similar to the

$c^{2} H$ stability criterion in the three-body problem. The Szebehely constant $C_{0}=-\frac{c^{2} E}{G^{2} M^{5}}$ is a function of the total energy $(E)$ and the magnitude of the angular momentum of the system (c), where $G$ is the gravitational constant, and $M$ is the total mass. The Szebehely constant can be determined by the initial conditions of the system.

One of the main difficulties in the study of the CSFBP is the existence of collision singularities which may cause numerical-integration algorithms to fail during a close encounter. It is also important to be able to compute the trajectories of the CSFBP orbits with a high order of accuracy, especially when studying the hierarchical evolution of systems (Széll et al. 2004a). Regularisation of two-body collisions by the Kustaanheimo-Stiefel method is an efficient mathematical tool for handling these kinds of singularities. Celletti (2002), Waldvogel (2008), and Aarseth (2003) provide detailed overviews of standard regularisation techniques in the N-body problem.

In this paper, we develop a suitable regularisation scheme for the collision singularities in the CSFBP and examine its performance. An outline of this regularisation scheme was briefly sketched in Sivasankaran et al. (2009). Here we provide complete details of the regularisation approach and present results from numerical testing of the algorithm.

In Sect. 2, we describe the important features of the CSFBP and derive its equations of motion. In Sect. 3, numerical investigations illustrate the unstable energy profile of the non-regularised equations at close encounters as well as indicate the rich structure of the binary interactions in the CSFBP. In Sect. 4, we develop the new regularisation scheme for the CSFBP and describe a method for the practical implementation of the regularisation algorithm. Section 5 presents numerical results comparing integration with and without regularisation. We illustrate the effectiveness of the new algorithm with different types of CSFBP orbits. We conclude in Sect. 6.

\section{An overview of the Caledonian symmetrical four-body problem}

In this section, we discuss the main features of the CSFBP [cf. Steves and Roy (2001)]. The coplanar CSFBP involves two pairs of distinct masses moving in coplanar, initially circular orbits, starting in a collinear arrangement. There are two types of symmetrical restrictions: past-future symmetry so that the dynamical evolution of the system after time $t=0$ is a mirror image of that before $t=0$; and dynamical symmetry for which the dynamical evolution of two bodies on one side of the system's centre of mass is a rotational image of that of the two bodies on the other side of the centre of mass. 
Fig. 1 a The initial configuration of the CSFBP $(t=0)$.

b The CSFBP configuration at a later time $(t>0)$ (a)

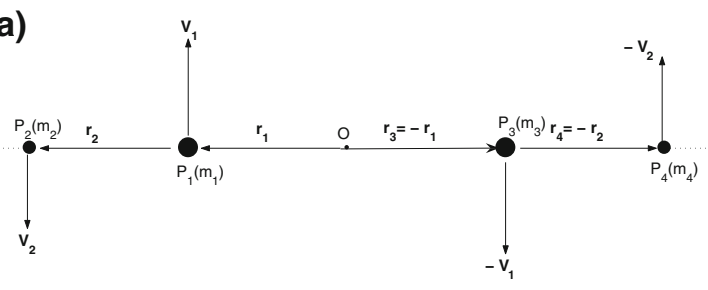

(b)

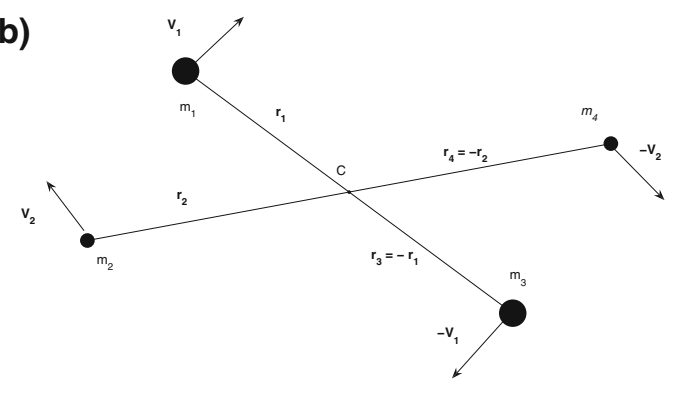

The initial configuration of the CSFBP is shown in Fig. 1. The four masses in the model $m_{1}, m_{2}, m_{3}, m_{4}$ are, respectively, at positions $P_{1}, P_{2}, P_{3}, P_{4}$ and interact under their mutual Newtonian gravitational attraction. We let $\mathbf{r}_{i}$ and $\dot{\mathbf{r}}_{i}$ be the radius and velocity vectors of the bodies with respect to the centre of mass of the four-body system, where $i=1,2,3,4$. The centre of mass itself is denoted by $O$. The CSFBP symmetrical restriction can be characterised by the following conditions:

1. The bodies at positions $P_{1}$ and $P_{3}$ have the same mass and likewise those at $P_{2}$ and $P_{4}$ have the same mass

$$
m_{1}=m_{3}, \quad m_{2}=m_{4} .
$$

2. The bodies initially at $P_{1}$ and $P_{3}$ move symmetrically with respect to each other about the centre of mass of the system. There is a similar symmetry for those initially at $P_{2}$ and $P_{4}$. Thus

$$
\mathbf{r}_{1}=-\mathbf{r}_{3}, \quad \mathbf{r}_{2}=-\mathbf{r}_{4}, \quad \mathbf{V}_{1}=\dot{\mathbf{r}}_{1}=-\dot{\mathbf{r}}_{3}, \quad \mathbf{V}_{2}=\dot{\mathbf{r}}_{2}=-\dot{\mathbf{r}}_{4} .
$$

This ensures dynamical symmetry for all time $t$. The resulting configuration is always a parallelogram. See Fig. $1 b$.

3. Initially, at time $t=0$, the bodies are collinear and have velocity vectors perpendicular to this line. Thus the past-future symmetry is obtained and

$$
\mathbf{r}_{1} \times \mathbf{r}_{2}=0, \quad \mathbf{r}_{1} \cdot \dot{\mathbf{r}}_{1}=0, \quad \mathbf{r}_{2} \cdot \dot{\mathbf{r}}_{2}=0 .
$$

We redefine the masses in terms of ratios to total mass: the total mass of the system $M$ is

$$
M=2\left(m_{1}+m_{2}\right),
$$

and we set

$$
\mu_{1}=\frac{m_{1}}{M}, \quad \mu_{2}=\frac{m_{2}}{M}, \quad \mu=\frac{\mu_{1}}{\mu_{2}}
$$

so that

$$
2\left(\mu_{1}+\mu_{2}\right)=1 .
$$


Taking the centre of mass to be at rest at the origin and the motion to lie in the $x y$-plane, the position coordinates of the four bodies can be taken to be $\mathbf{r}_{1}=\left(x_{1}, x_{2}\right), \mathbf{r}_{2}=$ $\left(x_{3}, x_{4}\right), \mathbf{r}_{3}=\left(-x_{1},-x_{2}\right), \mathbf{r}_{4}=\left(-x_{3},-x_{4}\right)$, with corresponding momenta $\left(\omega_{1}, \omega_{2}\right)=$ $\mu_{1} M\left(\dot{x_{1}}, \dot{x_{2}}\right),\left(\omega_{3}, \omega_{4}\right)=\mu_{2} M\left(\dot{x_{3}}, \dot{x_{4}}\right),\left(-\omega_{1},-\omega_{2}\right),\left(-\omega_{3},-\omega_{4}\right)$. With the symmetries present in the model, the Hamiltonian is

$$
\begin{aligned}
H= & \frac{1}{\mu_{1} M}\left(\omega_{1}^{2}+\omega_{2}^{2}\right)+\frac{1}{\mu_{2} M}\left(\omega_{3}^{2}+\omega_{4}^{2}\right) \\
& -2 G \mu_{1} \mu_{2} M^{2}\left(\frac{1}{r_{12}}+\frac{1}{r_{14}}\right)-\frac{G \mu_{1}^{2} M^{2}}{r_{13}}-\frac{G \mu_{2}^{2} M^{2}}{r_{24}},
\end{aligned}
$$

where the inter-body distances are

$$
\begin{aligned}
& r_{12}=\left(\left(x_{1}-x_{3}\right)^{2}+\left(x_{2}-x_{4}\right)^{2}\right)^{1 / 2}=r_{34}, \\
& r_{14}=\left(\left(x_{1}+x_{3}\right)^{2}+\left(x_{2}+x_{4}\right)^{2}\right)^{1 / 2}=r_{23}, \\
& r_{13}=\left(\left(2 x_{1}\right)^{2}+\left(2 x_{2}\right)^{2}\right)^{1 / 2}, \\
& r_{24}=\left(\left(2 x_{3}\right)^{2}+\left(2 x_{4}\right)^{2}\right)^{1 / 2} .
\end{aligned}
$$

Henceforth, for simplicity, we will set the gravitational constant $G$ and total mass $M$ to be equal to unity.

For initial conditions, we assume that the bodies start at $t=0$ on the $x$-axis, with velocities in the $y$-direction. So the initial coordinates are of the form $P_{1}\left(x_{1}, 0\right), P_{2}\left(x_{3}, 0\right), P_{3}\left(-x_{1}, 0\right)$, $P_{4}\left(-x_{3}, 0\right)$, with momenta $\left(0, \omega_{2}\right),\left(0, \omega_{4}\right),\left(0,-\omega_{2}\right),\left(0,-\omega_{4}\right)$.

Four types of two-body close encounters can occur in the CSFBP. Figure 2 illustrates these

1. "12"-type double-binary collision: collisions occurring in the binary formed between $P_{1}$ and $P_{2}$ and the symmetrical binary formed between $P_{3}$ and $P_{4}$ (Fig. 2 a).

2. "14"-type double-binary collision: collisions occurring in the binary formed between $P_{1}$ and $P_{4}$ and the symmetrical binary formed between $P_{2}$ and $P_{3}$ (Fig. 2b).

3. "13"-type single-binary collision: collision occurring in the binary formed between $P_{1}$ and $P_{3}$ (Fig. 2c).

4. "24"-type single-binary collision: collision occurring in the binary formed between $P_{2}$ and $P_{4}$ (Fig. 2d).

Fig. 2 The possible types of collisions present in the CSFBP; a "12"-type double binary, b "14"-type double binary, c "13"-type single binary, d "24"-type single binary (a)

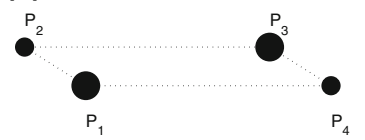

(c)

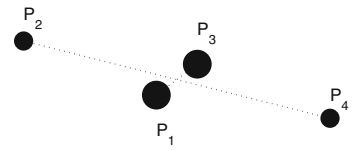

(b)

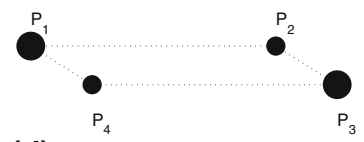

(d)

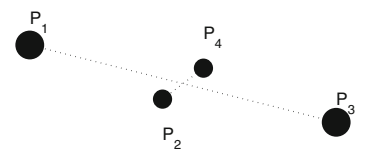




\section{Numerical investigations using the non-regularised equations of motion}

Hamilton's equations corresponding to the Hamiltonian (4),

$$
\frac{d x_{i}}{d t}=\frac{\partial H}{\partial \omega_{i}}, \quad \frac{d \omega_{i}}{d t}=-\frac{\partial H}{\partial x_{i}}, \quad i=1,2,3,4,
$$

can be directly integrated using a high-order numerical integrator. A variable step-size solver is advantageous as it adjusts the time steps automatically to maintain desired numerical accuracy, and thus it can handle moderately-close encounters efficiently. The Radau method of Everhart (1985) has been used, which is a 15th-order method with an adaptive step-size control and makes use of Gauss-Radau spacing. The Microsoft Visual Studio 2005 environment has been used for conducting the experiments. We set the error tolerance of the integrator to $10^{-12}$ and computations were performed in double precision.

As with earlier studies (Széll et al. 2004a,b), we consider systems with fixed values of the mass ratio $\mu$, initial energy $E$ and Szebehely constant $C_{0}$, and study the family of orbits arising from a grid of initial positions $\left(r_{1}, r_{2}\right)$, where $\left(r_{1}=x_{1}, r_{2}=x_{3}\right)$. The initial velocities are determined by these quantities. The orbital evolution is followed until either time $t=10^{5}$ integration time units is reached or else a close encounter has forced the termination of the trial. In the latter case the final type of two-body close encounter due to a breakdown in conservation of energy is recorded. Termination occurs whenever the numerical error in total energy from the initial energy becomes $\Delta E \geq 10^{-4}$.

Results from these simulations are shown in the form of an outcome chart, where each point in the grid of initial conditions is colour coded according to the nature of the final system. The outcome charts shown in Fig. 3 indicate that the majority of the regions of real motion are dominated by close encounters. In Széll et al. (2004b) fast-chaos detection methods were used to study the stable and chaotic behaviour of the CSFBP. Széll et al. (2004b) also recorded patterns of close encounters in the regions of real motion that are the same as those found in Fig. 3.

In the presence of close encounters, large energy errors occur in the non-regularised numerical integration and energy does not return to its pre-collision value after the encounter.
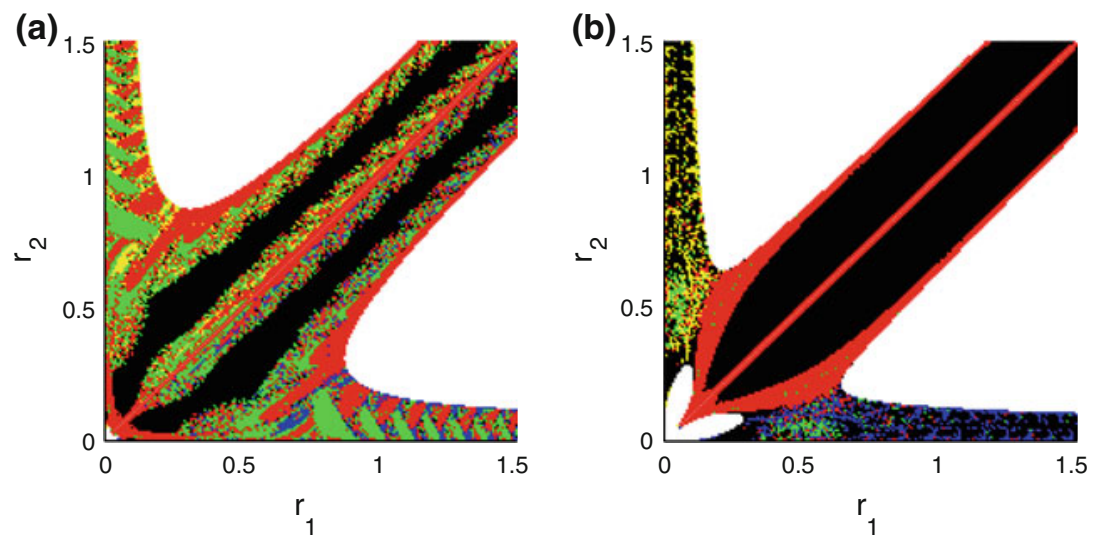

Fig. 3 Outcome charts, for $\mu=1, E=-7$, integration time $t=10^{5}$; a $C_{0}=10$, b $C_{0}=40$. Close encounters with break down of energy conservation are colour coded as follows: red "12" type, green "14" type, yellow "13" type, blue " 24 " type, or all grey shaded in the printed version. The black regions represent orbits where the systems were not terminated by close encounters 
Fig. 4 Energy profiles of two CSFBP close-encounter orbits integrated to time $t=20 \mathrm{using}$ the non-regularised equations of motion $(\mu=1, E=-7$ and $C_{0}=40$ as in Fig. 3b); solid line a "12"-type double-binary collision (initial $r_{1}=0.61$ and $\left.r_{2}=0.30\right)$; dotted line a "24"-type single-binary collision (initial $r_{1}=0.75$ and $r_{2}=0.01$ )

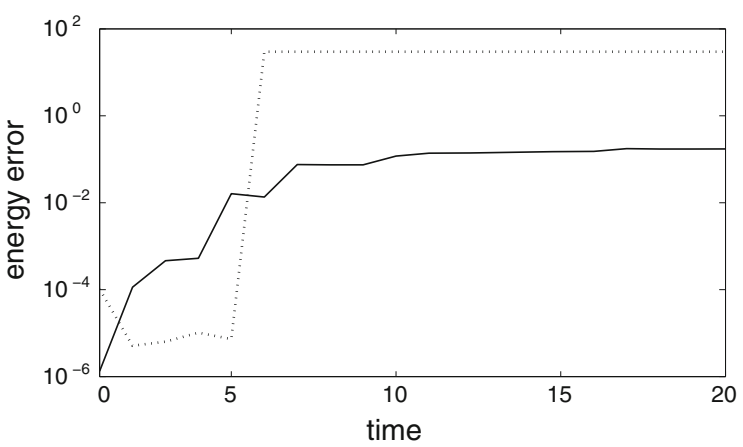

Figure 4 illustrates this behaviour in the numerical energy error for two individual closeencounter orbits. For both orbits, the energy fluctuation is mild until the close encounter. At that point, there is a rapid increase in energy error from which there is no return to the original energy.

In the next section, we present a global regularisation method for the two-body close encounters of the CSFBP.

\section{Regularisation procedure}

The regularisation scheme is an adaptation of the global regularisation scheme developed by D. C. Heggie (1974). The new scheme incorporates the symmetries of the CSFBP. All the distinct inter-body position vectors $\left(\mathbf{r}_{12}, \mathbf{r}_{14}, \mathbf{r}_{13}, \mathbf{r}_{24}\right)$ are regularised by the use of a LeviCivita type transformation. As a result of the process, the number of coordinates is doubled from four to eight. With a fixed centre of mass, the system is determined by the positions in the plane of just two bodies $m_{1}$ and $m_{2}$; however, there are four distinct inter-body vectors. A previous approach of this kind has been used for the simpler collinear symmetrical fourbody problem where only "12" and "13"-type binary collisions can occur (Sweatman 2002, 2006).

We introduce inter-body coordinates $q_{i}, i=1,2, \ldots, 8$ :

$$
\begin{aligned}
& \left(q_{1}, q_{2}\right)=\left(x_{1}, x_{2}\right)-\left(x_{3}, x_{4}\right), \\
& \left(q_{3}, q_{4}\right)=\left(x_{1}, x_{2}\right)+\left(x_{3}, x_{4}\right), \\
& \left(q_{5}, q_{6}\right)=2\left(x_{1}, x_{2}\right), \\
& \left(q_{7}, q_{8}\right)=2\left(x_{3}, x_{4}\right) .
\end{aligned}
$$

The corresponding conjugate momenta $p_{k}$ can be related to the original momenta $\left(\omega_{i}\right)$ by means of a generating function, $F\left(p_{k}, x_{i}\right)$ which is a function of two vectors $p_{k}$ and $x_{i}$ where $i=1-4$ and $k=1-8$. Thus,

$$
\omega_{i}=\frac{\partial F}{\partial x_{i}}, \quad F\left(p_{k}, x_{i}\right)=\sum_{j=1}^{8} p_{j} q_{j}\left(x_{i}\right) .
$$

However, a further four relations are required to determine the $p_{i}$ and so, analogous to the degeneracy found in the coordinates,

$$
\left(q_{5}, q_{6}\right)=\left(q_{1}, q_{2}\right)+\left(q_{3}, q_{4}\right), \quad\left(q_{7}, q_{8}\right)=\left(q_{3}, q_{4}\right)-\left(q_{1}, q_{2}\right) ;
$$


we set

$$
\left(p_{5}, p_{6}\right)=\left(p_{1}, p_{2}\right)+\left(p_{3}, p_{4}\right), \quad\left(p_{7}, p_{8}\right)=\left(p_{3}, p_{4}\right)-\left(p_{1}, p_{2}\right) .
$$

Using Eqs. (11) and (13), we deduce

$$
\begin{aligned}
& p_{1}=\frac{1}{6}\left(\omega_{1}-\omega_{3}\right), \quad p_{2}=\frac{1}{6}\left(\omega_{2}-\omega_{4}\right), \\
& p_{3}=\frac{1}{6}\left(\omega_{1}+\omega_{3}\right), \quad p_{4}=\frac{1}{6}\left(\omega_{2}+\omega_{4}\right), \\
& p_{5}=\frac{1}{3} \omega_{1}, \quad p_{6}=\frac{1}{3} \omega_{2}, \\
& p_{7}=\frac{1}{3} \omega_{3}, \quad p_{8}=\frac{1}{3} \omega_{4} .
\end{aligned}
$$

The system is transformed to Levi-Civita-type coordinates $\left(Q_{k}, P_{k}\right), k=1,2, \ldots, 8$ by setting

$$
q_{j}=Q_{j}^{2}-Q_{j+1}^{2}, \quad q_{j+1}=2 Q_{j} Q_{j+1},
$$

for $j=1,3,5,7$. The corresponding conjugate momenta $P_{k}$ are

$$
P_{k}=\frac{\partial F_{2}}{\partial Q_{k}}, \quad F_{2}\left(p_{k}, Q_{k}\right)=\sum_{j=1}^{8} p_{j} q_{j}\left(Q_{k}\right), \quad k=1, \ldots, 8
$$

that is

$$
P_{j}=2 p_{j} Q_{j}+2 p_{j+1} Q_{j+1}, \quad P_{j+1}=2 p_{j+1} Q_{j}-2 p_{j} Q_{j+1},
$$

for $j=1,3,5,7$. Following the method of Heggie (1974), we additionally introduce a rescaled time $\tau$ modified to best suit the CSFBP with symmetrical inter-body distances. We also choose, different from Heggie (1974), an exponent power of 5/2 which we found to produce the best performance in conserving energy.

$$
\begin{aligned}
\frac{d t}{d \tau} & =g=\frac{r_{12} r_{13} r_{14} r_{24}}{\left(r_{12}+r_{13}+r_{14}+r_{24}\right)^{5 / 2}} \\
& =\frac{\left(Q_{1}^{2}+Q_{2}^{2}\right)\left(Q_{3}^{2}+Q_{4}^{2}\right)\left(Q_{5}^{2}+Q_{6}^{2}\right)\left(Q_{7}^{2}+Q_{8}^{2}\right)}{\left(Q_{1}^{2}+Q_{2}^{2}+Q_{3}^{2}+Q_{4}^{2}+Q_{5}^{2}+Q_{6}^{2}+Q_{7}^{2}+Q_{8}^{2}\right)^{5 / 2}} .
\end{aligned}
$$

The final transformed Hamiltonian takes the form

$$
\Gamma\left(Q_{k}, P_{k}\right)=g\left(\tilde{H}-h_{0}\right)
$$

where $\tilde{H}\left(Q_{k}, P_{k}\right)$ is the original Hamiltonian $(H)$ considered as a function of the $Q_{k}$ and $P_{k}$, and $h_{0}$ is the initial (constant) value of $\tilde{H}$. The new regularised equations of motion are

$$
\begin{aligned}
\frac{d Q_{i}}{d \tau} & =\frac{\partial \Gamma}{\partial P_{i}}, \\
\frac{d P_{i}}{d \tau} & =-\frac{\partial \Gamma}{\partial Q_{i}} .
\end{aligned}
$$

Equations (18), (20) and (21) are integrated with respect to $\tau$ to find the trajectories.

The symbolic differentiation to derive the gradient of $\Gamma\left(Q_{i}, P_{i}\right)$ produces a large number of additive and multiplicative terms. To make the integrator efficient, we have optimised the 
regularised equations using a technique discussed in Gruntz and Waldvogel (1998), ie. we have used the reverse-mode algorithmic differentiation to reduce the total number of multiplicative operations (multiplication and addition). This technique enables the derivation of the partial derivatives of the regularised Hamiltonian $\Gamma\left(Q_{i}, P_{i}\right)$ using MAPLE.

There is still a singularity should all four bodies simultaneously collide. This is impossible for a CSFBP system with non-zero angular momentum. In general such four-body collisions cannot be regularised.

In the next section, we study the effectiveness of this global regularisation method for a range of CSFBP orbits and compare the performance with that of the non-regularised equations of motion.

\section{The performance of the regularisation method}

The numerical experiments as described in Sect. 3 were repeated using the regularised equations. The accuracy of the floating-point arithmetic and the user-defined error tolerance of the integration will also have an effect on the accuracy of the integration. We therefore keep these identical for both the regularised and non-regularised integrations to show the effect of regularisation on the accuracy. All the orbits shown in Fig. 3 which resulted in termination due to close encounters can now be successfully integrated through close encounters to the end of the simulation $\left(t=10^{5}\right)$ without breaching the energy error bound $\left(\Delta E \geq 10^{-5}\right)$.

We now further illustrate the numerical results with individual orbital trajectories of the CSFBP of the types: regular, irregular and escape.

\subsection{Regular orbit}

The first orbit selected is regular and quasi-periodic. This orbit does not contain any extreme close encounters as the masses remain well separated. Results from the (a) non-regularised and (b) regularised simulations are shown in Fig. 5.

The orbital trajectories in the $x y$-plane of motion are shown in Fig. 5I. Only the positions of masses $m_{1}\left(x_{1}, x_{2}\right)$ (green) and $m_{2}\left(x_{3}, x_{4}\right)$ (blue) are shown. The other two masses mirror their motion. The regularised and non-regularised numerical trajectories are similar.

Figure 5II compares the numerical energy errors over 50 time units. In both cases the energy profile appears stable with no large spikes, however, the regularised energy error is better on average by a factor of order 10 .

Figure 5III shows the step-sizes used during the integrations as a function of time. The Radau15 variable step-size solver automatically adjusts the step-sizes to maintain the prescribed tolerance level during integration. We set the error tolerance of the integrator to $10^{-12}$, for both the regularised and non-regularised orbits. The step-sizes with the regularisation procedure are less variable in both the relative magnitude of the variation compared with step-size and also in the frequency of the variation. The lower variability makes it easier for the regularised equations to be integrated, as fewer integration steps will fail and need to be repeated. Overall, aside from repeated steps, the time steps moved forward in physical time were larger for the regularised scheme and so the number of integration steps was reduced by a factor of 1.6.

The main motivation for the regularisation scheme described in this paper was to be able to determine the orbits that the integrator had previously failed on; however, even for the present orbit there are numerical advantages. For the same tolerance in the Radau integrator, there is both an improvement in energy conservation but also an improvement in CPU time 
I

(a)

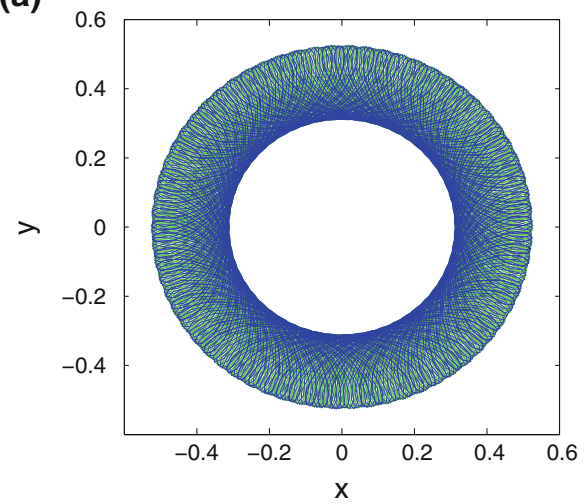

II

(a)

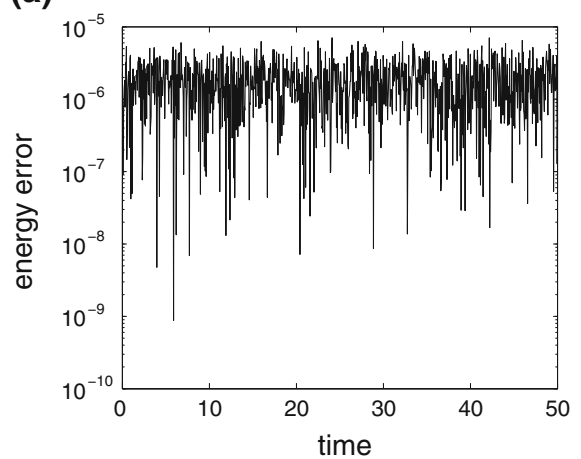

III

(a)

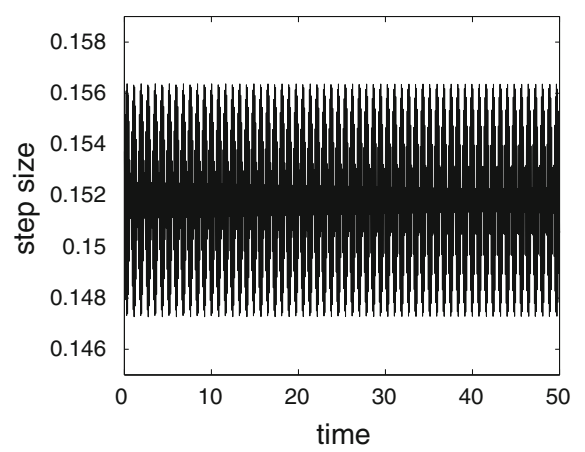

(b)

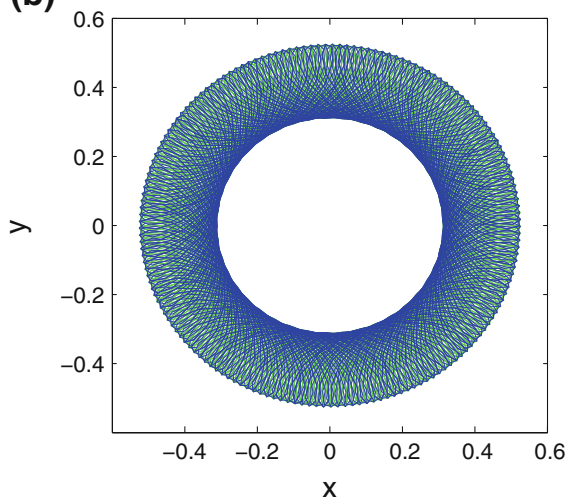

(b)

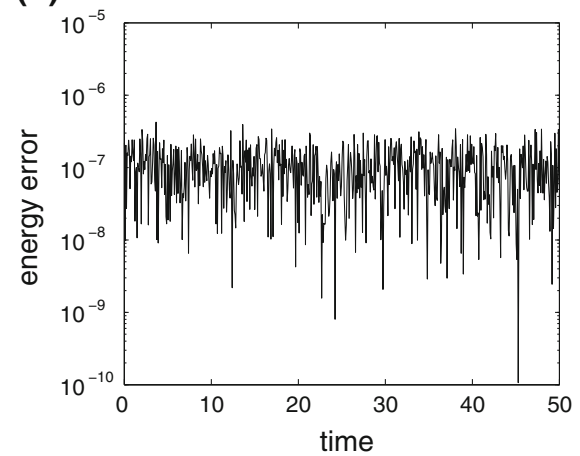

(b)

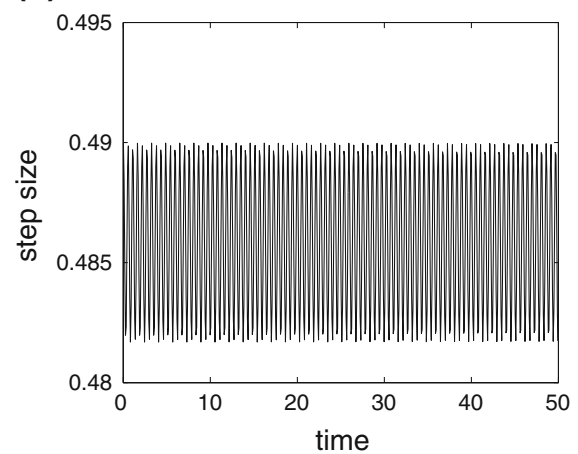

Fig. 5 A quasi-periodic orbit over the time $[0,50]\left(\mu=1, E=-7, C_{0}=10\right.$ initial $r_{1}=0.52121$ and $r_{2}=0.31082$ ); with a non-regularised; $\mathbf{b}$ regularised equations. I Trajectories of $m_{1}$ (green) and $m_{2}$ (blue) in the $x y$-plane of motion. In the printed version, green is light grey and blue is dark grey; II energy error; III time step variations (Note the ordinates have a different range) 
Fig. 6 Irregular orbits starting with the same initial conditions over the time period $[0,100]$ ( $\mu=1, E=-1.2, C_{0}=0.6$, initial $r_{1}=1.00001$ and $\left.r_{2}=0.50001\right)$; with $\mathbf{a}$ non-regularised; $\mathbf{b}$ regularised equations. I Trajectories of $m_{1}$ (green) and $m_{2}$ (blue) in the $x y$-plane of motion. In the printed version, green is light grey and blue is dark grey; II energy error over the time period $[0,400]$
I

(a)

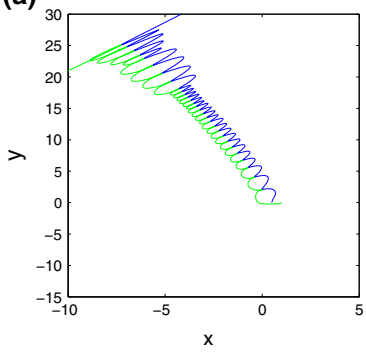

(b)

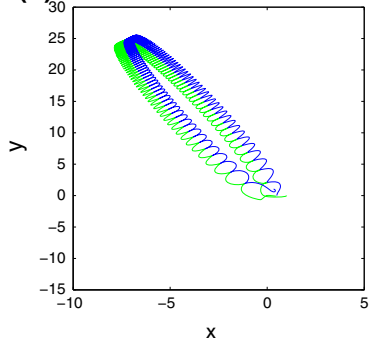

II

(a)

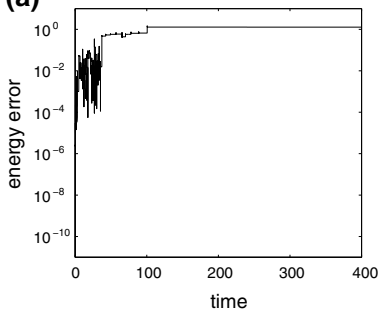

(b)

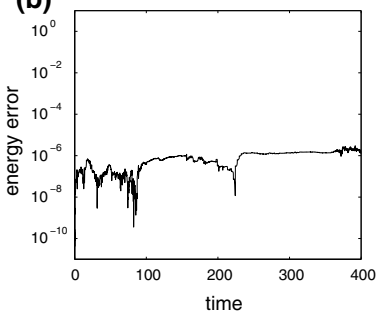

by a factor of 1.12 . The latter improvement occurs despite the regularised scheme requiring more than twice as many equations to be integrated and extra calculation being needed for the coordinate transformations back to physical coordinates.

\subsection{Irregular orbit}

The next orbit to be studied is irregular with several close encounters which test the capability of the regularised method to handle collisional events. We reduce the error tolerance of the integrator to $10^{-14}$ for both the regularised and non-regularised orbits in order to have an example where the non-regularised orbit has a chance of being accurate through the first few near-collision events. The two different realisations of the orbit in the $x y$-plane using non-regularised and regularised equations are contained in Fig. 6I. Both orbits begin the same with an interplay of "12"-type close encounters. (These are mirrored by the " 34 " symmetrical pair on the other side of the centre of mass, which is not shown in the figure.) Close-encounter events exist from the beginning of the CSFBP orbital evolution in both non-regularised and regularised cases. We can see that the first few close encounters (at $t=0.572,1.336$ and 3.366) have an important influence in deciding the trajectory of the CSFBP orbit. Figure 6II shows the energy error observed on the time interval $[0,400]$. The conservation of energy breaks down for the non-regularised integrator at the seccond close encounter at $t \approx 1.366$, with a corresponding energy error of the order $10^{-2}$. The numerically derived orbit is not reliable after this point; although the two orbits remain similar in appearance until the 22nd close encounter at $t \approx 37.313$. At this time, the orbit becomes highly irregular with an energy error of the order $10^{-1}$ (See Fig. 6I.a, II.a). The numerical orbit computed with regularisation is well behaved with an energy error of the order of maximum $10^{-6}$ and survives the interplay of close two-body encounters between the " 12 " binaries efficiently. 
Fig. 7 An escape orbit to $t=10^{3} ; \mu=1, E=-1$ and $C_{0}=0.3$ with $r_{1}=45, r_{2}=42.9$, initially. The initial velocities are \pm 0.0009 in the transverse direction

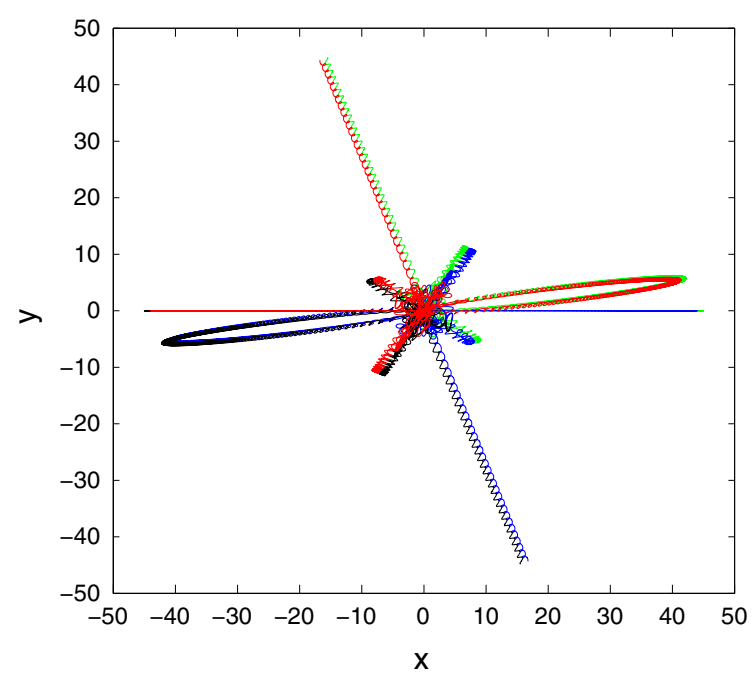

Fig. 8 Energy profile of the escape orbit shown in Fig. 7 until time $t=10^{4}$

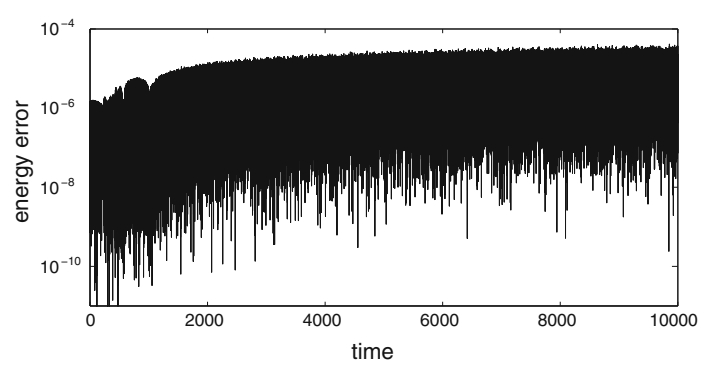

\subsection{Escape orbit}

The third orbit to be studied illustrates the energy-conserving capability of our regularisation scheme in the presence of extreme close-encounter events leading to escape. The four masses are initially far from the origin with very small initial velocities. Only the regularised integration of the orbit is shown. The corresponding non-regularised integration of these initial conditions is unreliable with the energy error growing exponentially with time from the first close encounter at $t \approx 0$. Figure 7 , shows the escape orbit over $10^{3}$ time units. The positions of all the masses are shown in the $x y$-plane of motion. Masses $m_{1}, m_{2}, m_{3}$ and $m_{4}$ are denoted by green, blue, black and red lines, respectively. This CSFBP system starts as two binary-subsystems ("12" and "34"), followed by a series of close-encounter events before the final escape of two binary-subsystems ("14" and " 23 "). Figure 8 shows the corresponding well-controlled energy profile for $t=10^{4}$ integration time and illustrates the long-term reliability of the regularised scheme.

\section{Concluding remarks}

A global regularisation scheme for numerically integrating a symmetrically restricted fourbody problem, the Caledonian symmetric four-body problem (CSFBP), has been presented. 
This regularisation consists of several Levi-Civita-type coordinate transformations together with a time transformation that will remove the singularities due to colliding pairs of masses. The new approach has been tested upon a range of CSFBP orbits and demonstrates a good performance with close encounters.

Acknowledgments For useful suggestions and discussions regarding numerical implementation of the regularised codes, the authors thank András Széll and Mohammed Shoaib.

\section{References}

Aarseth, S.J.: Gravitational N-Body Simulations. Cambridge University Press, London (2003)

Celletti, A.: Singularities, collisions and regularisation theory. In: Benest, D., Froeschlé, C. (eds.) Singularities in Gravitational Systems, pp. 25-50. Springer, Berlin (2002)

Everhart, E.: An efficient integrator that uses Gauss-Radau spacings. In: Caruci, A., Valsecchi, G.B. (eds.) The Dynamics of Comets: Their Origin and Evolution, pp. 185-202. Reidel, Dordrecht (1985)

Gruntz, D., Waldvogel, J.: Orbits in the planar three-body problem. In: Gander, W., Hrebicek, J. (eds.) Solving Problems in Scientific Computing Using Maple and Matlab, pp. 51-72. Springer, New York (1998)

Heggie, D.C.: A global regularisation of the gravitational N-body problem. Celest. Mech. Dyn. Astron. 10, $217-$ 241 (1974)

Loks, A., Sergysels, R.: Zero velocity hypersurfaces for the general planar four-body problem. Astron. Astrophys. 149, 462-464 (1985)

Marchal, C., Saari, D.G.: Hill regions for the general three-body problem. Celest. Mech. Dyn. Astron. 12, $115(1975)$

Roy, A.E., Steves, B.A.: Some special restricted four-body problems-II: from Caledonian to Copenhagen. Planet. Space Sci. 46, 1475-1486 (1998)

Roy, A.E., Steves, B.A.: The Caledonian symmetrical double binary four-body problem: surfaces of zero velocity using the energy integral. Celest. Mech. Dyn. Astron. 78, 299-318 (2001)

Sekiguchi, M., Tanikawa, K.: On the symmetric collinear four-body problem. Publ. Astron. Soc. Jpn. 56, 236251 (2004)

Sergysels, R., Loks, A.: Restrictions on the motion in the general four-body problem. Astron. Astrophys. 182, 163-166 (1987)

Sivasankaran, A., Steves, B.A., Sweatman, W.L.: Close encounters in the Caledonian symmetric four-body problem. In: Varvoglis, H., Knežević, Z. (eds.) Proceedings of the International Conference on the Dynamics of Celestial Bodies, Greece, June 2008, pp. 177-180. Aristotle University, Thessaloniki and Astronomical Observatory, Belgrade (2009)

Steves, B.A., Roy, A.E.: Some special restricted four-body problems-I: modelling the Caledonian problem. Planet. Space Sci. 46, 1465-1474 (1998)

Steves, B.A., Roy, A.E. : Surfaces of separation in the Caledonian symmetrical double binary four-body problem. In: Steves, B.A., Maciejewski, A.J. (eds.) The Restless Universe: Application of Gravitational N-body Dynamics to Planetary, Stellar and Galactic Systems, pp. 301-325. IOP Publishing, Bristol (2001)

Sweatman, W.L.: The symmetrical one-dimensional Newtonian four-body problem: a numerical investigation. Celest. Mech. Dyn. Astron. 82, 179-201 (2002)

Sweatman, W.L.: A family of Schubart-like interplay orbits and their stability in the one-dimensional four-body problem. Celest. Mech. Dyn. Astron. 94, 37-65 (2006)

Széll, A., Steves, B.A., Érdi, B.: The hierarchical stability of quadruple stellar and planetary systems using the CSFBP model. Astron. Astrophys. 427, 1145-1154 (2004a)

Széll, A., Érdi, B., Sándor, Z., Steves, B.A.: Chaotic and stable behavior in the Caledonian symmetric four-body problem. MNRAS 347, 380-388 (2004b)

Waldvogel, J.: Quaternions for regularizing celestial mechanics: the right way. Celest. Mech. Dyn. Astron. 102, $149-162(2008)$

Zare, K.: The effects of integrals on the totality of solutions of dynamical systems. Celest. Mech. Dyn. Astron. 14, 73-83 (1976)

Zare, K.: Bifurcation points in the planar problem of three bodies. Celest. Mech. Dyn. Astron. 16, 35-38 (1977) 